Article

\title{
Immunization with E Plus NS1-2a Enhanced Protection against Dengue Virus Serotype 2 in Mice
}

\author{
Yanhua Wu ${ }^{1}$, Shuyu Fang ${ }^{1}$, Xiaoyun Cui, Na Gao, Dongying Fan, Jing An * \\ Department of Microbiology, School of Basic Medical Sciences, Capital Medical University, \\ Beijing, 100069, China; wuyanhua@ccmu.edu.cn; fangshuyu_2007@126.com; cxy9567@sina.com; \\ gao_na@ccmu.edu.cn; dengue@ccmu.edu.cn \\ ${ }^{1}$ First co-author, Yanhua Wu and Shuyu Fang contributed equally to this work \\ * Corresponding author, E-mail addresses: anjing@ccmu.edu.cn; Tel.: +86 1083950107
}

\begin{abstract}
Dengue virus (DENV), the causative agent of dengue fever (DF), is one of the most important mosquito-borne viruses that can infect humans. Although much effort has been made on prevention and control of dengue, there are currently no anti-viral drugs or worldwide approved vaccines yet. In this study, we immunized six-week-old Balb/c mice with DNA vaccine candidates E and NS1-2a of DENV serotype 2 or the combination of them (E+NS1-2a) via an electroporation (EP)-assisted intramuscular gene delivery system and evaluated the immune response and protection. The highest specific antibody titres and cytokine levels secreted by splenocytes as well as the highest survival rate were observed in the E+NS1-2a group, followed by E group and NS1-2a group. Our data suggested that the combination of E and NS1-2a delivered by EP may be a superior preventive strategy against DENV.
\end{abstract}

Keywords: Dengue virus; E; NS1-2a; electroporation; DNA vaccine

\section{Introduction}

Dengue viruses (DENVs) are members of the flaviviridae family and a major cause of arthropod-borne diseases worldwide. DENVs are composed of four serotypes (DENV1-4) and the infection with any serotype could cause classical dengue fever (DF) and/or severe dengue including dengue haemorrhagic fever (DHF) and dengue shock syndrome (DSS). The viruses are spread in tropical and subtropical areas of the world [1] with dramatically grown global incidence in recent years. It is estimated that there are 390 million dengue infections per year, of which 96 million manifest clinically [2], a rate is three times higher than that reported in 2009. Notably, in 2014, the Guangdong province (south of China) erupted in the most serious dengue epidemic in history, with more than 45,000 DF reported cases. Therefore, DF is one of the fastest-growing health burdens and has been a severe public health problem. However, there are no worldwide certified vaccines or specific antivirals available currently. Last year, a recombinant live-attenuated tetravalent dengue vaccine (CYD-TDV, ChimeriVax) produced by Sanofi Pasteur has been licensed in Mexico and other countries [3]. The CYD-TDV vaccine was asymmetrically protective. However, a recent study reported vaccine-related antibody-dependent enhancement (ADE), which causes younger children 
and seronegative individuals an increased risk of developing a severe case during subsequent DENV infections [4]. Thus, it is still urgent to develop a safer, more effective and costless dengue vaccine.

DNA vaccines have been proved to trigger long-lasting humoral and cellular immunity $[5,6]$; and therefore were considered to be a suitable choice for developing a dengue vaccine. Despite much effort has been made on research of DNA vaccines for decades, there is still no human DNA vaccine available in clinic, mainly due to the fact that naked DNA can be quickly degraded by DNases before uptake by host cells [7], which leads to a low immune response [6]. To resolve this issue, several strategies were recently developed, and the EP gene delivery system has been shown to enhance uptake and expression of DNA in host cells [8-11].

The genome of DENV is a single-stranded positive-sense RNA that encodes three structural proteins (the capsid protein $\mathrm{C}$, the precursor of membrane protein $\mathrm{prM}$, and the envelope protein $\mathrm{E}$ ) and seven non-structural proteins (NS1 to NS5). Of those, the E is the major structural protein exposed on the surface of the virion and contains the most important neutralizing epitopes. NS1 is the unique glycoprotein among seven non-structural proteins. It can not only be expressed on the infected cell surface, but also secreted into patient serum. Furthermore, antibodies against NS1 can interact with complement protein, which lyse the infected cell by antibody-dependent cell-mediated cytotoxicity (ADCC)[12]. It is widely accepted that the E gene is the main molecular target for developing flavivirus DNA vaccines. However, effect of NS1 in protection against DENVs remains controversy[13-17]. Previously we have demonstrated that recombinant plasmids expressing $\mathrm{E}$ and/or NS1 could induce immune responses when inoculate to mice with intramuscular (IM) injection with the varied survival rates (30\% to 40\%)[18], indicating a limited protection. The present study aimed to further evaluate the immunogenicity and protection of plasmids expressing E or NS1 or their combination through EP in mice. Our results suggested that the combined immunization of E+NS1-2a elicited an effective immune response and protection and EP is an effective way to deliver DNA vaccine.

\section{Materials and Methods}

\subsection{Cells, virus and mice}

Aedes albopictus mosquito cells (C6/36) were grown at $28^{\circ} \mathrm{C}$ in RPMI1640 media containing $10 \%$ fetal bovine serum (FBS, Gibco, Auckland, New Zealand). Vero cells were cultivated at $37^{\circ} \mathrm{C}$ in minimal essential medium (MEM, Invitrogen, USA) supplemented with 5\% FBS.

Six-week-old Balb/c mice were purchased from the Academy of Military Medical Sciences (Beijing, China) and were housed in a pathogen-free environment. The animals were manipulated according to "the animal experiments and experimental animal administrative regulations of Capital Medical University of China". The protocol was approved by Comments of Animal Experiments and Experimental Animal Welfare Committee of Capital Medical University of China (approval number AEEI-2015-048).

DENV2 (strain TR1751) was propagated in Aedes albopictus mosquito (C6/36) cells, and viral 
titres were detected by plaque assay using Vero cell monolayer under $1.2 \%$ methyl cellulose.

\subsection{Construction and identification of plasmids}

E (from 877 to $2421 \mathrm{bp}$ ) and NS1-2a (from 2388 to $4131 \mathrm{bp}$ ) genes were amplified from DENV2-infected C6/36 cells by PCR, and the primers were listed in Table 1 . The genes were constructed in the vector pReceiverM01 and were named pRe/E and pRe/NS1-2a. The recombinant plasmids were identified by sequence analysis (Sangon Biotech Co., Ltd., Shanghai, China) and the expression of the proteins was confirmed by indirect immunofluorescence (IFA)[19]. In brief, plasmids were transfected into Vero cells by Lipofectamine 2000 (Invitrogen, California, USA). At $24 \mathrm{~h}$ after transfection, the cells were fixed with $4 \%$ polyformaldehyde and permeabilized by $0.2 \%$ Triton X-100. After washing and blocking, the cells were incubated with monoclonal anti-DENV2 E or NS1 antibody (Abcam, Tokyo, Japan) at $4^{\circ} \mathrm{C}$ overnight. Then, the cells were incubated with FITC-conjugated goat anti-mouse IgG (Earthox, San Francisco, USA). The results were analysed by fluorescent microscope (Olympus, Japan).

Table 1. Constructions of plasmids of DENV2

\begin{tabular}{|c|c|c|c|}
\hline Recombinant plasmids & Amplified regions & Primers & Restriction sites \\
\hline \multirow[t]{2}{*}{$\mathrm{pRe} / \mathrm{E}$} & $E(877-2421)$ & P1:5 'TTCGAAATGACACATTTCCAAAGAG3' & $N_{s p \mathrm{~V}}$ \\
\hline & & P2:5' CTCGAGGGCCTGCACCATAACTCC $3^{\prime}$ & XhoI \\
\hline \multirow[t]{2}{*}{$\mathrm{pRe} / \mathrm{NS} 1-2 \mathrm{a}$} & NS1-2a (2337-4131) & P1:5' CGTTCGAATCATGAACTCACGTAGCACTT $3^{\prime}$ & $N_{s p \mathrm{~V}}$ \\
\hline & & P2:5'ATAGCGGCCGCCTACCTTTTCTTACTGGTTCTTG3' & Not $\mathrm{I}$ \\
\hline
\end{tabular}

\subsection{Immunization and challenge experiments in mice}

The 6-week-old Balb/c mice were divided into 4 groups (20 mice for each group): E, NS1-2a, E+NS1-2a, and pReceiverM01 groups. Mice were inoculated with E $(50 \mu \mathrm{g})$ or NS1-2a $(50 \mu \mathrm{g})$ or

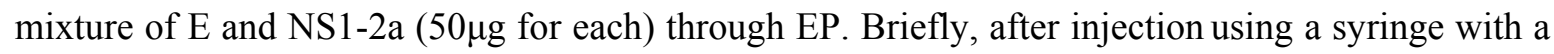
25-27 gauge needle, two silver electrodes ( $6 \mathrm{~mm}$ apart) were immediately inserted the injection site bilaterally, and six electric pulses $(36 \mathrm{~V}, 10 \mathrm{~ms})$ were applied by a gene delivery device (Terasa Healthcare Sci-Tech, China). Mouse immunized with 50 $\mu$ g of pReceiverM01 served as a control. Three weeks after the final immunization, 6 mice in each group were euthanized for collecting sera and spleen samples to evaluate the humoral and cellular immunity. The other 14 mice were intracerebrally (i.c.) injected with a lethal dose (50LD50) of DENV2 using a 27-gauge one-stop needle (Top Injection Needle, Tokyo, Japan). These mice were monitored daily, and the morbidity and mortality was evaluated for three weeks. Clinical symptoms were scored as follows: 0: healthy; 1: ruffled hair; 2: hunchbacked appearance; 3: bradykinesia; 4: paralysis; and 5: death. 


\subsection{Antibody assay by enzyme-linked immunosorbent assay (ELISA)}

Mouse serum samples were collected by tail-bleeding three weeks after the final immunization, and the antibodies against DENV2 were evaluated using ELISA as described previously[19]. Briefly, 96-well microtiter plates were coated with $5 \mu \mathrm{g}$ of concentrated DENV2 protein as the antigens. $100 \mu 1$ of two-fold serial dilutions of the serum samples were added to each well. After washing with $0.05 \%$ Tween-20 in Phosphate Buffered Saline (PBS-T), the plates were incubated with goat anti-mouse IgG-horseradish peroxidase (HRP) (1:3000, KPL, USA) at $37^{\circ} \mathrm{C}$ for $1 \mathrm{~h}$, followed by addition of the orthophenylenediamine substrate solution for visualization. The reactions were stopped by addition of $2 \mathrm{M} \mathrm{H}_{2} \mathrm{SO}_{4}$. The absorbance was measured at $492 \mathrm{~nm}$ with a microplate reader (Multiskan, Mk3, Thermo, USA). The end-point titres of the serum samples were determined as the reciprocal of the highest dilution, giving an optical density (OD) twice that of the non-immune serum. End-point titres of anti-DENV2 antibodies were recorded as geometrical mean titres (GMT). To determine the IgG subclass, antibody isotype ELISAs were performed, in which anti-mouse IgG-HRP was replaced with anti-mouse IgG1-HRP or anti-mouse IgG2a-HRP (BD PharmingenTM, USA).Values of IgG subclasses were reported as the mean \pm SD of the OD at $492 \mathrm{~nm}$ at a serum dilution of 1:400.

\subsection{Cytokine ELISPOT assays}

Three weeks after the final vaccination, splenocytes were isolated by mouse lymphocyte separation medium (DAKEWEI, Beijing China), and the production of IFN- $\gamma$, IL-2, IL-4, and IL-10 were measured using an ELISPOT assay according to the manufacturer's instructions (BD Biosciences, USA) [20]. Briefly, 96-well filtration plates (Millipore, Bedford, USA) were pre-coated with anti-mouse IFN- $\gamma$, IL-2, IL-4 or IL-10 antibodies, and then $1 \times 10^{6}$ splenocytes were added to each well. After treatment with concentrated DENV2 $(50 \mu \mathrm{g} / \mathrm{ml})$, ConA $(5 \mu \mathrm{g} / \mathrm{ml}$, Sigma, positive control) or RPMI 1640 medium alone (negative control), biotin-conjugated antibodies were added (streptavidin-HRP). The spot-forming units (SFU), representing positive cells, were counted automatically using a CTL ELISpot reader (CTL, USA) and analysed using ImmunoSpot image analyser software v4.0.

\subsection{Statistical analysis}

All data were analysed with SPSS software (version 17.0). Significant difference between groups was calculated using one-way ANOVA and a survival Kaplan Meier analysis. The results were considered to be significant at $p<0.05(*)$ and highly significant at $p<0.01(* *)$. 


\section{Results}

\subsection{The recombinant plasmids efficiently expressed in eukaryotic cells}

To test the expression of the recombinant plasmids $p R e / E$ and pRe/NS1-2a in eukaryotic cells, plasmid DNA was transfected into Vero cells, and IFA was performed. As shown in Figure 1., specific fluorescence was observed in the cytoplasm of the transfected-Vero cells, while no specific fluorescent signals were detected in control cells transfected with pReceiverM01. This indicated that all recombinant plasmids could be efficiently expressed in eukaryotic cells and can be used in subsequent experiments.

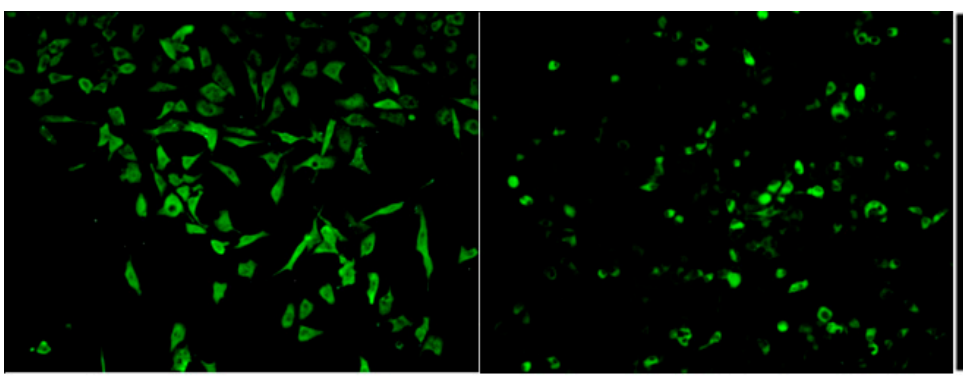

A

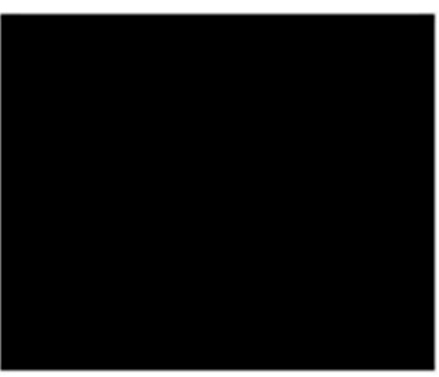

C

Figure 1. The expression of recombinant plasmids in Vero cells.

$\mathrm{pRe} / \mathrm{E}$ or $\mathrm{pRe} / \mathrm{NS} 1-2 \mathrm{a}$ was transfected into Vero cells, and expression was analysed with anti-DENV2 E or NS1 antibody. $\mathrm{A}, \mathrm{B}$ and $\mathrm{C}$ : Expression of $\mathrm{pRe} / \mathrm{E}$ or $\mathrm{pRe} / \mathrm{NS} 1-2 \mathrm{a}$ or control plasmid pReceiverM01 in Vero was analysed with anti-DENV2 E antibody (A) or anti-DENV2 NS1 antibody (B) or mixture of these two antibodies ( C).

\subsection{Antibody response to immunizations}

Three weeks after the final immunization, serum samples were collected for determining anti-DENV2 antibody levels. As expected, the mice inoculated with E, NS1-2a or E+NS1-2a showed increasing end-point titres of anti-DENV2 IgG compared with the control group (pReceiverM01, $P<0.01$ ). Of those, the E+NS1-2a group showed the highest anti-DENV2 antibody titres, with a GMT of 1:9051, while antibody titres in the NS1-2a and E groups were 1:800 or 1:3200, respectively (Figure 2.). These results suggested that our DNA vaccine candidates could induce effective immune responses against DENV2. Levels of anti-DENV2 IgG1 and IgG2a were also detected, and results were shown in Figure 3.. Both IgG1 and IgG2a increased in all the groups compared to the control group. But there were some differences among these groups: in E group IgG2a titre was higher than IgG1 $(p<0.05)$ with significantly increased $\operatorname{IgG} 2 \mathrm{a} / \mathrm{IgG} 1$ ratio, which showed unconspicuous change in NS-2a and $\mathrm{E}+\mathrm{NS} 1-2 \mathrm{a}$ groups. Interestingly, the obviously increased IgG1 level was observed in the $\mathrm{E}+\mathrm{NS} 1-2 \mathrm{a}$ group compared with that in $\mathrm{E}$ group $(\mathrm{P}<0.05)$ (Figure 3.). The results indicated that different immune response biases were induced by our DNA vaccine candidates, which express different immunogens and may be linked to the protective efficacy. 


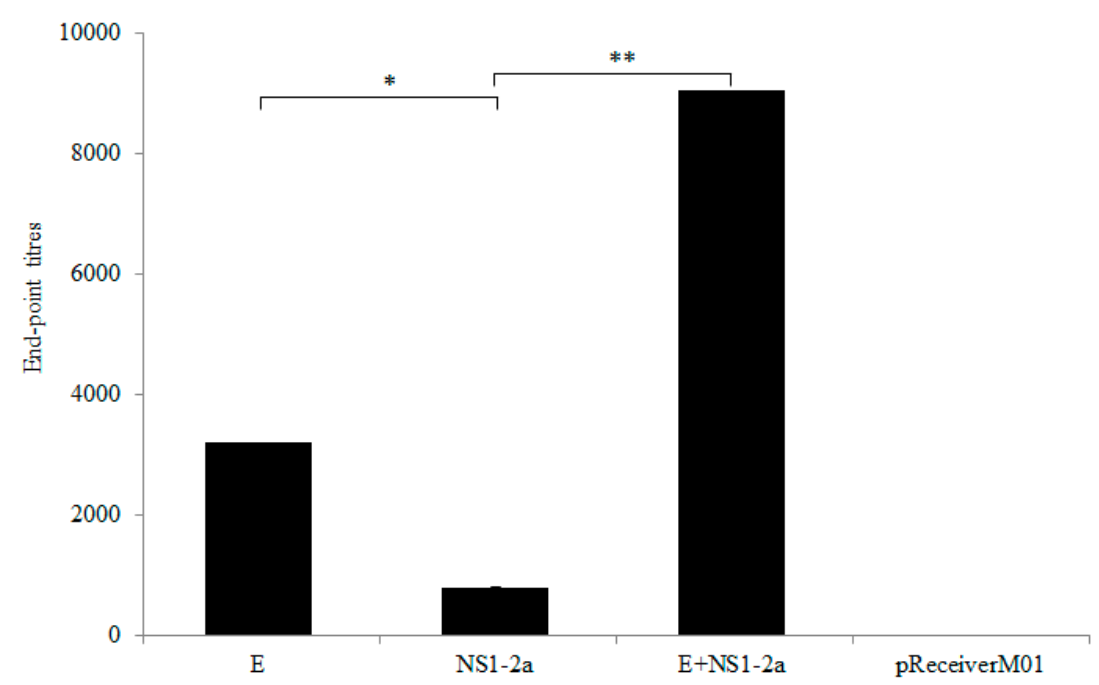

Figure 2. End-point titres of anti-DENV2 antibodies in plasmids-immunized mice (Mean $\pm \mathrm{SD}$ ).

Mouse serum samples were collected three weeks after the final immunization, and the antibodies against DENV2 were evaluated by ELISA. The end-point titres in serum samples were determined as the reciprocal of the highest dilution, giving an optical density (OD) value twice that of the non-immune serum from control mice. The bar graph shows the geometrical mean standard deviation (SD) values for all the groups $(n=6)$ $\left({ }^{*} \mathrm{p}<0.05 ; * * \mathrm{p}<0.01\right.$, one-way ANOVA test).

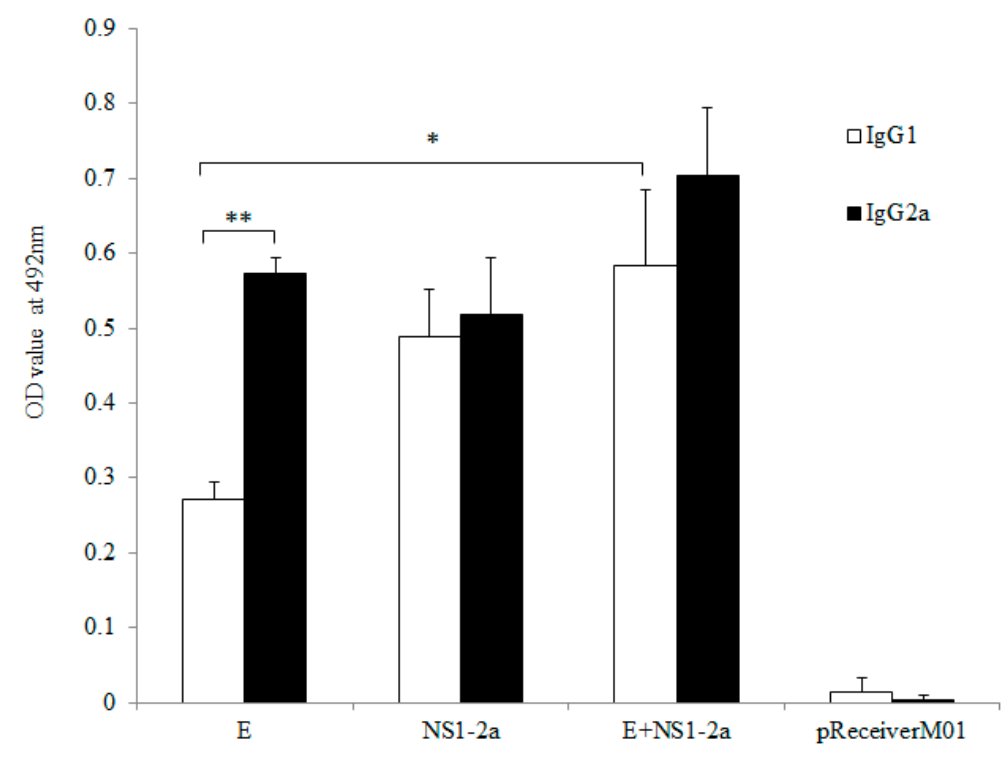

Figure 3. Determination of DENV2-specific serum IgG subclasses in each group (Mean $\pm \mathrm{SD}, \mathrm{n}=6$ ). Antigen-specific ELISA is reported as the OD values at 492nm at a serum dilution of 1:400. The bar graph shows the mean SD values for OD values of all the groups with plasmids $\left({ }^{*} \mathrm{p}<0.05 ; * * \mathrm{p}<0.01\right.$, one-way ANOVA test).

\subsection{Generation of cytokines in the plasmid-immunized mice}

Cytokines play a crucial role in the induction and modulation of the immune responses. The levels of cytokines secreted by splenocytes of immunized mice upon stimulation with the DENV2 
antigen were evaluated by ELISPOT. As shown in Figure 4., all cytokines tested, including IFN- $\gamma$, IL-2, IL-4 and IL-10, showed a similar change pattern: the cytokines increased significantly in the $\mathrm{E}+\mathrm{NS} 1-2 \mathrm{a}$ and $\mathrm{E}$ groups $(\mathrm{P}<0.01$ or 0.05$)$ and showed no obvious change in the NS1-2a group compared with control group. It is known that IFN- $\gamma$ and IL-2, as well as IL-4 and IL-10, act as markers of the Th1 and the Th2 immune responses, respectively. These results suggested that both antibody and cell-mediated immune responses were induced and they may contribute to protection against DENV2 infection.
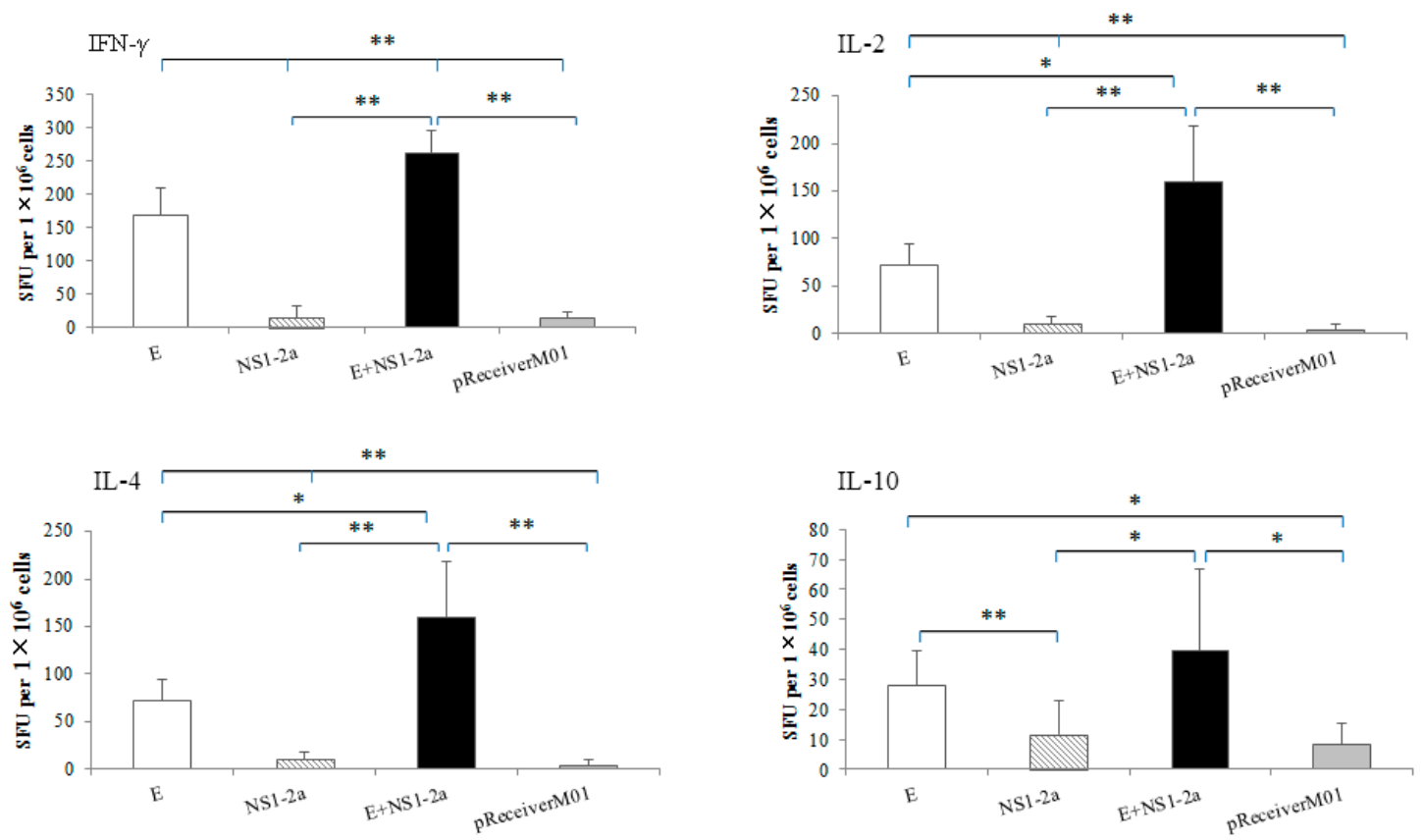

Figure 4. Splenocyte-secreted IFN- $\gamma$, IL-2, IL-4 and IL-10 upon DENV2 antigen stimulation were detected by ELISPOT assays (mean $\pm \mathrm{SD})$.

Splenocytes were collected from mice $(n=6)$ three weeks after the final immunization. The numbers of IFN- $\gamma(A)$, IL-2(B), IL-4(C), and IL-10 (D) positive cells are recorded as the mean spot-forming units (SFU) $/ 10^{6}$ splenocytes $+\operatorname{SD}\left({ }^{*} \mathrm{p}<0.05\right.$; $* * \mathrm{p}<0.01$, one-way ANOVA test).

\subsection{Protection against DENV2 challenge}

The protective efficacy was evaluated by challenge with a lethal dose of DENV2 in vaccinated animals and clinical symptoms and mortality rate were recorded for 21 days after the challenge. As shown in Figure 5., mice in the control group showed severe illness symptoms such as paralysis and moribundity and finally died. In contrast, mice in E+NS1-2a group only had slight and transient clinical signs such as ruffled hair appearance and hypokinesia and then gradually recovered. Mice in NS1-2a and E groups had asthenia, wasting or bradykinesia, indicating moderate severity of the illness compared with other groups (Figure 5.A). Accordingly, all mice in the control group died at day 13 after challenge. In contrast, the highest survival rate of $85.7 \%$ was observed in the 
E+NS1-2a group (12/14), followed by 57.1\% (8/14) in E group and 50\% (7/14) in NS1-2a (Figure 5.B). The survival rates in E and E+NS1-2a groups showed significant differences from that in the control group $(\mathrm{P}<0.01$ or 0.05$)$.
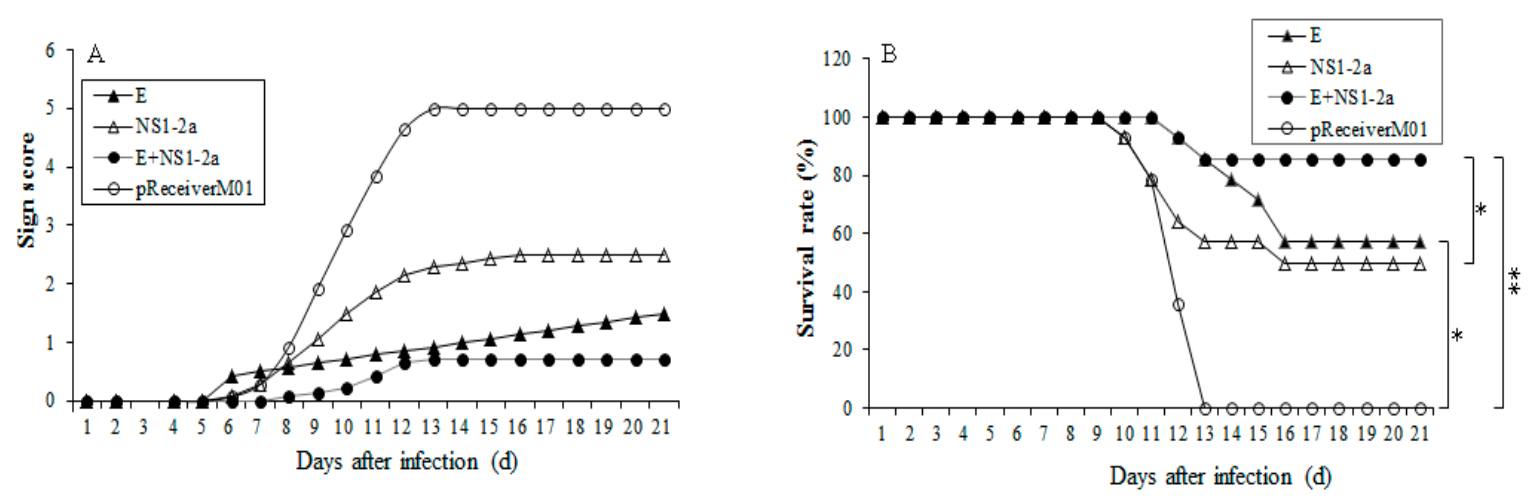

Figure 5. Survival rate and sign score of mice immunized with plasmids and challenged with 50 LD50 of DENV2 $(n=14)$

Mice were challenged with DENV2 three weeks after the final immunization and the morbidity and mortality was evaluated for three weeks. Clinical symptoms were scored as follows: 0: healthy; 1: ruffled hair; 2: hunchbacked appearance; 3: bradykinesia; 4: paralysis; and 5: death. A. Sign score of mice immunized with plasmids and challenged with 50 LD50 of DENV2. Pathological symptoms are recorded as the mean sign scores.B. Survival rate of mice immunized with plasmids and challenged with 50 LD50 of DENV2. The survival rate was shown as the percentage of survivors.

\section{Discussion}

Dengue is an acute systemic viral disease that has established itself globally in both endemic and epidemic transmission cycles. Although the recombinant vaccine CYD-TDV has currently been licensed, it was reported that the vaccine increase severity of disease in younger children and seronegative individuals, which may due to the unbalanced immune response against all four DENV serotypes [4]. Moreover, a one-year inoculation period of the vaccine may limit the administration of the full course in travellers [21]. DNA vaccines have been proved to confer many advantages such as costless, high safety and long-term persistence of immunogens and holds promise for use in humans. DNA immunization has been widely employed in development of several vaccines since the 1990s and currently four veterinary DNA vaccines for horses, dogs, pigs and fish have been licensed [22]. However, there is no effective human DNA vaccine available due to the poor immunogenicity observed in clinical studies. Our previous studies and others showed that co-expressing prME and NS1 of DENV1 or DENV2 could induce strong immune responses and enhance survival of infected mice $[18,23,24]$ when compared with prME alone. However, the long fragment of prME and NS1 may limit DNA expression and thereby reduce immune response. Recently, we have proved that EP could enhance DNA-uptake and expression in mice and pigs $[10,11]$. Thus, to obtain effective immune responses and protection against DENV2, E and NS1 proteins were utilized to immunize mice via EP in this study. Compared with E or NS1-2a alone, 
high levels of anti-DENV2 antibodies and cytokines as well as good protection against DENV2 challenge were found in mice immunized with E+NS1-2a, indicating that E+NS1-2a via electroporation is effective way for prevention of DENV infection.

Two factors were considered to contribute the effective protection in this study. We think that the first important one is the immunogens used in the experiment. Dengue E protein contains the most important neutralizing antigenic sites, and antibodies against E protein can neutralize the same serotype of DENV and block the infection. NS1 protein, the only non-structural glycoprotein expressed on the cell surface, is secreted in patient serum and can invoke an immune response and confer protection in animals by ADCC without the adverse effects of ADE [25]. In this study, E and NS1-2a were used separately or in combination and the characterization of immune responses induced by these two proteins showed some differences: in E group, the IgG2a levels were higher than the IgG1 levels $(\mathrm{P}<0.01)$ and all tested cytokines increased while the NS1-2a induced an equally increased level of both IgG1 and IgG2a and unchanged cytokine levels. This indicated that two proteins induced different immune response bias, which may be link to the limited protection observed in these two groups. The E+NS1-2a could produce elevated IgG1 and IgG2a titres, obviously increased cytokine (IFN- $\gamma$, IL-2, IL-4 and IL-10) levels and effective protection, indicating the importance of combined use of the two proteins in inducing balanced Th1 and Th2 type immune responses and the prevention. Furthermore, E+NS1-2a induced the highest anti-DENV titre, indicating there was no interference in expression and inducing immune response between those two proteins, implying that two proteins may trigger immune response via different mechanism. Our results were further supported by study from Gonçalves group, which found that effective defences against DENV can be achieved with the combination of both antibodies and $\mathrm{T}$ cells responses but not any of them alone [24]. Our results suggested that a combination of E and NS1-2a could induce both humoral and cellular immunity and that a balanced Th1/Th2 immune response is a primary goal for the development of DENV vaccines.

Next, effective immunity induced by E+NS1-2a should owe to EP delivery system. It is known that EP transiently creates pores in the myocyte membranes and then effectively enhances the cellular uptake of plasmids, which dramatically affects the magnitude of the immune responses. It was reported that DNA vaccines have been less efficient and limited protection in larger animal models, even at multiple immunizations and high DNA doses. However, our recent study demonstrated that elevated expression of the prME protein was observed in EP-administered mice and rabbits[11], and higher levels of specific IgG and neutralizing antibody were also successfully induced. It suggested that EP may circumvent the barrier, raise transfection efficiency and internalization and substantially elevate the potency of DNA vaccines and enhance immune protection. Taken together, our results suggested that a combination of E and NS1-2a may be a novel strategy against DENV infection and EP may be the inspiring gene delivery strategy in DNA vaccine development. 


\section{Acknowledgments}

This study was supported by the Natural Science Foundation of China (No. 81471957, 81401676 and 81271839), Beijing Natural Science Foundation (7144194) and Scientific Research Common Program of Beijing Municipal Commission of Education (SQKM201210025005)

\section{Author Contributions}

Yanhua Wu, Shuyu Fang, Xiaoyun Cui, Na Gao, and Dongying Fan performed the experiments; Yanhua $\mathrm{Wu}$ and Shuyu Fang analyzed the data and wrote the paper; Jing An designed the experiments, wrote and revised the paper.

\section{Conflicts of Interest}

All the authors declare no conflict of interest. The founding sponsors had no role in the design of the study; in the collection, analyses, or interpretation of data; in the writing of the manuscript, and in the decision to publish the results.

\section{References}

1. Simasathien, S.; Watanaveeradej, V. Dengue vaccine. J Med Assoc Thai 2005, 88 Suppl 3, S363-377.

2. Bhatt, S.; Gething, P.W.; Brady, O.J.; Messina, J.P.; Farlow, A.W.; Moyes, C.L.; Drake, J.M.; Brownstein, J.S.; Hoen, A.G.; Sankoh, O., et al. The global distribution and burden of dengue. Nature 2013, 496, 504-507.

3. Recker, M.; Vannice, K.; Hombach, J.; Jit, M.; Simmons, C.P. Assessing dengue vaccination impact: Model challenges and future directions. Vaccine 2016, 34, 4461-4465.

4. Halstead, S.B.; Russell, P.K. Protective and immunological behavior of chimeric yellow fever dengue vaccine. Vaccine 2016.

5. Hansen, S.G.; Ford, J.C.; Lewis, M.S.; Ventura, A.B.; Hughes, C.M.; Coyne-Johnson, L.; Whizin, N.; Oswald, K.; Shoemaker, R.; Swanson, T., et al. Profound early control of highly pathogenic siv by an effector memory t-cell vaccine. Nature 2011, 473, 523-527.

6. Dong, Y.; Yang, J.; Zhang, J.; Zhang, X. Nano-delivery vehicles/adjuvants for DNA vaccination against hiv. J Nanosci Nanotechnol 2016, 16, 2126-2133.

7. Wijesundara, D.K.; Jackson, R.J.; Ramshaw, I.A.; Ranasinghe, C. Human immunodeficiency virus-1 vaccine design: Where do we go now? Immunol Cell Biol 2011, 89, 367-374.

8. Tollefsen, S.; Vordermeier, M.; Olsen, I.; Storset, A.K.; Reitan, L.J.; Clifford, D.; Lowrie, D.B.; Wiker, H.G.; Huygen, K.; Hewinson, G., et al. DNA injection in combination with electroporation: A novel method for vaccination of farmed ruminants. Scand J Immunol 2003, 57, 229-238.

9. Zhao, Y.G.; Peng, B.; Deng, H.; Chen, G.; Yang, F.; Shao, M.; Lu, H.; Li, Y.; Peng, J.; Xu, L., et al. Anti-hbv immune responses in rhesus macaques elicited by electroporation mediated DNA vaccination. Vaccine 2006, 24, 897-903.

10. Sheng, Z.; Gao, N.; Cui, X.; Fan, D.; Chen, H.; Wu, N.; Wei, J.; An, J. Electroporation enhances protective immune response of a DNA vaccine against japanese encephalitis in mice and pigs. Vaccine 2016, 34, 5751-5757.

11. Chen, H.; Zheng, X.; Wang, R.; Gao, N.; Sheng, Z.; Fan, D.; Feng, K.; Liao, X.; An, J. Immunization with electroporation enhances the protective effect of a DNA vaccine candidate expressing prme 
antigen against dengue virus serotype 2 infection. Clin Immunol 2016, 171, 41-49.

12. Schlesinger, J.J.; Foltzer, M.; Chapman, S. The fc portion of antibody to yellow fever virus ns1 is a determinant of protection against yf encephalitis in mice. Virology 1993, 192, 132-141.

13. Henchal, E.A.; Henchal, L.S.; Schlesinger, J.J. Synergistic interactions of anti-ns1 monoclonal antibodies protect passively immunized mice from lethal challenge with dengue 2 virus. J Gen Virol 1988, 69 ( Pt 8), 2101-2107.

14. Qu, X.; Chen, W.; Maguire, T.; Austin, F. Immunoreactivity and protective effects in mice of a recombinant dengue 2 tonga virus ns1 protein produced in a baculovirus expression system. $J$ Gen Virol 1993, 74 ( Pt 1), 89-97.

15. Puttikhunt, C.; Kasinrerk, W.; Srisa-ad, S.; Duangchinda, T.; Silakate, W.; Moonsom, S.; Sittisombut, N.; Malasit, P. Production of anti-dengue ns1 monoclonal antibodies by DNA immunization. $J$ Virol Methods 2003, 109, 55-61.

16. Schlesinger, J.J.; Brandriss, M.W.; Walsh, E.E. Protection of mice against dengue 2 virus encephalitis by immunization with the dengue 2 virus non-structural glycoprotein ns1. J Gen Virol 1987, 68 ( Pt 3), 853-857.

17. Costa, S.M.; Paes, M.V.; Barreto, D.F.; Pinhao, A.T.; Barth, O.M.; Queiroz, J.L.; Armoa, G.R.; Freire, M.S.; Alves, A.M. Protection against dengue type 2 virus induced in mice immunized with a DNA plasmid encoding the non-structural 1 (ns1) gene fused to the tissue plasminogen activator signal sequence. Vaccine 2006, 24, 195-205.

18. Lu, H.; Xu, X.F.; Gao, N.; Fan, D.Y.; Wang, J.; An, J. Preliminary evaluation of DNA vaccine candidates encoding dengue- 2 prm/e and ns1: Their immunity and protective efficacy in mice. Mol Immunol 2013, 54, 109-114.

19. Chen, H.; Gao, N.; Fan, D.; Wu, J.; Zhu, J.; Li, J.; Wang, J.; Chen, Y.; An, J. Suppressive effects on the immune response and protective immunity to a jev DNA vaccine by co-administration of a gm-csf-expressing plasmid in mice. PLoS One 2012, 7, e34602.

20. Li, J.; Chen, H.; Wu, N.; Fan, D.; Liang, G.; Gao, N.; An, J. Characterization of immune responses induced by inactivated, live attenuated and DNA vaccines against japanese encephalitis virus in mice. Vaccine 2013, 31, 4136-4142.

21. Flipse, J.; Smit, J.M. The complexity of a dengue vaccine: A review of the human antibody response. PLoS Negl Trop Dis 2015, 9, e0003749.

22. Kutzler, M.A.; Weiner, D.B. DNA vaccines: Ready for prime time? Nat Rev Genet 2008, 9, 776-788.

23. Zheng, Q.; Fan, D.; Gao, N.; Chen, H.; Wang, J.; Ming, Y.; Li, J.; An, J. Evaluation of a DNA vaccine candidate expressing prm-e-ns1 antigens of dengue virus serotype 1 with or without granulocyte-macrophage colony-stimulating factor (gm-csf) in immunogenicity and protection. Vaccine 2011, 29, 763-771.

24. Goncalves, A.J.; Oliveira, E.R.; Costa, S.M.; Paes, M.V.; Silva, J.F.; Azevedo, A.S.; Mantuano-Barradas, M.; Nogueira, A.C.; Almeida, C.J.; Alves, A.M. Cooperation between cd4+ t cells and humoral immunity is critical for protection against dengue using a DNA vaccine based on the ns1 antigen. PLoS Negl Trop Dis 2015, 9, e0004277. 
25. Mellado-Sanchez, G.; Garcia-Cordero, J.; Luria-Perez, R.; Lazaro-Olan, L.; Santos-Argumedo, L.; Gutierrez-Castaneda, B.; Estrada-Garcia, I.; Cedillo-Barron, L. DNA priming e and ns1 constructs--homologous proteins boosting immunization strategy to improve immune response against dengue in mice. Viral Immunol 2005, 18, 709-721.

(C) 2016 by the authors; licensee Preprints, Basel, Switzerland. This article is an open access article distributed under the terms and conditions of the Creative Commons by Attribution (CC-BY) license (http://creativecommons.org/licenses/by/4.0/). 